\title{
Letter by Garcia A, et al. regarding article: Coronary artery disease risk reclassification by a new acoustic-based score
}

\author{
Artemio García-Escobar ${ }^{1}\left[\right.$ · Alfonso Martín-Martínez ${ }^{2} \cdot$ Jorge Del-Toro-Cervera $^{3} \cdot$ Pablo Demelo-Rodríguez $^{3}$
}

Received: 6 December 2019 / Accepted: 7 January 2020 / Published online: 26 February 2020

(c) Springer Nature B.V. 2020

\begin{abstract}
The best cost-effective non-invasive test for the diagnosis of coronary artery disease (CAD) in patients with intermediate pre-test probability (PTP) is unknown. Nevertheless one of the most common non invasive test used is the exercise treadmill testing (ETT) that is the less expensive non-invasive test but with the lowest sensitivity for the diagnosis of CAD, therefore many patients with intermediate PTP will required another non-invasive test with a higher cost and some of them require exposure to radiation. Despite all these measures, an estimated $\$ 108.9$ billion is spent annually on CAD treatment. Some studies had showed that diastolic dysfunction is associated to CAD. A novel signal-processed surface ECG (MyoVista hsECG) can detection the abnormal myocardial relaxation and therefore identified CAD. The non-invasive acoustic device CADScore V3 algorithm had lower cost compared with any noninvasive test, with a high negative predictive value but not good enough specificity to diagnose $\mathrm{CAD}$, hence should be the first approach in patients with a low and intermediate probability of CAD, and if to this evaluation will added the Myovista hs ECG to detection of CAD, therefore some patients with intermediate PTP could be reclassified into high risk and a better cost-effective decisions could be taken as referring directly to coronary angiography.
\end{abstract}

Keywords Coronary artery disease $\cdot$ Non-invasive acoustic device $\cdot$ Diastolic disfunction $\cdot$ Cadscore $\cdot$ Cadence $\cdot$ Noninvasive test $\cdot$ Pre-test probability of coronary artery disease $\cdot$ CAD consortium clinical score $\cdot$ MyoVista hsECG

We congratulate to Schmidt et al. for the non-invasive acoustic device CADScor ${ }^{\circledR}$ System Acarix A/S with the CADScore V3 algorithm to reclassify patients with intermediate pre-test probability (PTP) and clinically suspected stable coronary artery disease (CAD) into a low probability group thereby ruling out significant CAD [1].

Evaluation of individuals without known CAD presenting with stable chest pain syndrome is most cost-effective when exercise treadmill testing (ETT) is performed first,

This comment refers to the article available online at https://doi. org/10.1007/s10554-019-01746-y.

Artemio García-Escobar

dr_garciaescobar@hotmail.com

1 Cardiology Department, Hospital Universitario Severo Ochoa, Madrid, Spain

2 Emergency Department, Hospital Universitario Severo Ochoa, Madrid, Spain

3 Internal Medicine Department, Hospital General Universitario Gregorio Marañón, Madrid, Spain followed by imaging tests for ETT that is equivocal or not able to be performed. In individuals up to high likelihood of significant CAD, stress echocardiography (SE) or coronary computed tomographic angiography (CCTA) represent the most cost-effective strategies [2]. Despite all these measures, an estimated $\$ 108.9$ billion is spent annually on CAD treatment [3].

The ETT is a less expensive non-invasive test but with the lowest sensitivity for the diagnosis of CAD, therefore many patients with intermediate PTP will require another noninvasive test with a higher cost and some of them require exposure to radiation (see Table 1).

The SCOT-HEART Coronary CTA Cohort study $\mathrm{n}=1738$, compared 3 risk scores for estimating PTP of CAD, the updated Diamond-Forrester score (UDF), CAD Consortium clinical score (CAD2) and Confirm risk score (CRS). The discrimination of each score was assessed by area under the receiver-operating curve (AUC) and reclassification by net reclassification improvement (NRI) to identify low-risk patients at the $10 \%$ probability. The CAD2 provided the best discrimination (CAD2 AUC 0.79, UDF AUC 
Table 1 Non-invasive tests used to diagnose coronary artery disease

\begin{tabular}{llll}
\hline Non-invasive test & Sensitivity (\%) & Specificity (\%) & References \\
\hline Exercise treadmill testing (ETT) & $45-50$ & $85-90$ & {$[18]$} \\
Stress echocardiography (SE) & 82 & 84 & {$[19]$} \\
Coronary computed tomography angiography (CCTA) & 85 & 90 & {$[20]$} \\
Single-photon emission computed tomography (SPECT) & 83 & 77 & {$[21]$} \\
Vasodilator stress positron emission tomography (PET) & $81-97$ & $74-91$ & {$[22]$} \\
Vasodilator stress magnetic resonance imaging & $67-94$ & $61-85$ & {$[22]$} \\
\hline
\end{tabular}

0.77, CRS AUC 0.75, $\mathrm{p}<0.001$ for both comparisons) and also the best reclassification (CAD2 NRI 0.31, CRS NRI 0.21 vs UDF, $\mathrm{p}<0.001$ for all comparisons) [4].

Some studies had shown that diastolic dysfunction is associated with CAD [5]. Hoffmann et al. showed that patients with significant CAD compared with those without significant CAD had more abnormal echocardiographic diastolic parameters. After multivariable analysis tissue Doppler echocardiography (TDE) parameters of early diastolic velocity ( $\left.\mathrm{e}^{\prime}\right)$ and systolic velocity ( $\left.\mathrm{s}^{\prime}\right)$ remained independent predictors of $\mathrm{CAD}, \mathrm{e}^{\prime}$ odds ratio (OR) $1.5(1.1-1.9, \mathrm{p}<0.01)$ per $\mathrm{cm} / \mathrm{s}$ decrease and s' OR $1.7(1.1-2.5, \mathrm{p}<0.05)$ per $\mathrm{cm} / \mathrm{s}$ decrease. AUC for ETT in combination with TDE was significantly higher than AUC for ETT alone (0.84 vs 0.79, p<0.01) [6]. Hwang et al. demonstrated in patients with heart failure with preserved ejection fraction (HFpEF) the $68 \%$ had angiographicallyproven CAD [7]. HFpEF is defined by left ventricular ejection fraction (LVEF) $\geq 50$ percent and the dominant abnormality resides in diastole [8]. Therefore early recognition of left ventricular diastolic performance alteration may signal the presence of CAD.

Recently some electrocardiographic (ECG) novel signs for detection of diastolic dysfunction has come out. The QT interval is measured from the beginning of the QRS complex to the end of the $\mathrm{T}$ wave and approximates the time it takes the ventricles to repolarize. Long QT syndrome (LQTS) is the prolongation of the QT interval. LQTS may be either congenital or acquired [9]. The National Health and Nutrition Examination Survey III $(\mathrm{n}=8561)$ showed that risk factors for acquired prolonged QTc interval were the age, female sex, hypocalcemia, hypokalemia, history of thyroid disease, history of myocardial infarction and taking QTprolonging medications [10]. LQTS has traditionally been considered to be purely electrical, but mechanical alterations in patients with LQTS were reported. Leren et al. Showed in patients with genotyped LQTS compared with healthy controls had a lower systolic function by global longitudinal strain (GLS), longer contraction duration and reduced diastolic function [11]. Disturbed diastolic function occurs with impaired sarcoplasmic reticulum calcium handling as occurs in myocardial ischemia, hypertension or diabetes [12]. Thus, the delayed uptake of calcium is pathophysiologically associated with prolongation of QT interval [13]. Wilcox et al. Showed that QTc was inversely associated with TDE $\mathrm{e}^{\prime}$ with a correlation coefficient $(\mathrm{R})=-0.54(\mathrm{p}<0.0001)$, using a cut-off value of QTc $\geq 435 \mathrm{~ms}$ had a sensitivity $73 \%$ and specificity $74 \%$ for detection of diastolic dysfunction, when restricted to patients with known or suspected heart failure (HF) had a sensitivity $80 \%$ and specificity $90 \%$ [14].

Another diastolic dysfunction ECG sign is the T-peak to T-end interval (TpTe). Sauer et al. demonstrated that increased $\mathrm{TpTe}$ was inversely associated with reduced TDE $e^{\prime}(\mathrm{R}=-0.66, \mathrm{p}<0.0001)$ and used a cut-off value of $\mathrm{TpTe} \geq 75 \mathrm{~ms}$ for detection of abnormal diastolic stress test (Peak exercise E/e' $>13$ ) [15]. Moreover, Namdar et al. showed that T-peak to $\mathrm{P}$ wave (Tend-P) interval using cutoff value of $\leq 311 \mathrm{~ms}$ had a sensitivity $79 \%$ and specificity $72 \%$ for detection of diastolic dysfunction, but using a novel index calculated as Tend-P interval/PQ interval $\times$ age using cut-off value of $\geq 0.0333$ had a AUC 0.91 , sensitivity $82 \%$ and specificity $93 \%$ for detection of diastolic dysfunction [16]. Many physicians are unaware of those ECG signs, and most of these signs require exact manual measurements that can take time. Sengupta et al. used a novel signal-processed surface ECG algorithm to extract electrophysiological signal patterns uniquely associated with abnormal myocardial relaxation (MyoVista hsECG Informatics, HeartSciences, Southlake, Texas), showing an AUC 0.91, sensitivity 80\% and specificity $84 \%$ for prediction of low $\mathrm{e}^{\prime}$ velocity (septal $\mathrm{e}^{\prime}<7 \mathrm{~cm} / \mathrm{s}$ and/or lateral $<10 \mathrm{~cm} / \mathrm{s}$ ), low e' velocity also correctly identified concomitant significant CAD in the $82 \%$ [17].

CADScore ${ }^{\circledR}$ System and CADenceTM Ironman are the non-invasive acoustic device approved by the CE (Conformité Européenne), CADenceTM Ironman also is approved by the FDA (Food and Drug Administration). These noninvasive acoustic devices are feasible, easy to perform at the office visit or bedside, lower cost compared with any noninvasive test, with a high negative predictive value but not good enough specificity to diagnose CAD. The pre-test probability of CAD should be calculated using the CAD2 clinical score. Definitely the non-invasive acoustic devices should be the first approach in patients with a low and intermediate probability of CAD and patients with low risk for UA. If to this evaluation will add the signal-processed surface ECG 
algorithm (MyoVista hsECG) to the detection of abnormal myocardial relaxation and therefore identified CAD, hence some patients with intermediate PTP can be reclassified into high risk, and a better cost-effective decision could be taken as referring directly to coronary angiography.

\section{References}

1. Schmidt SE et al (2019) Coronary artery disease risk reclassification by a new acoustic-based score. Int J Cardiovasc Imaging 35(11):2019-2028

2. Min JK et al (2017) Cost-effectiveness of diagnostic evaluation strategies for individuals with stable chest pain syndrome and suspected coronary artery disease. Clin Imaging 43:97-105

3. Heindenreich PA, Trogdon JG, Khavjou OA et al (2011) Forecasting the future of cardiovascular disease in the United States: a policy statement from the American Heart Association. Circulation 123(8):933-944

4. Baskaran L et al (2019) A Comparison of the Updated DiamondForrester, CAD Consortium, and CONFIRM History-Based Risk Scores for Predicting Obstructive Coronary Artery Disease in Patients With Stable Chest Pain: The SCOT-HEART Coronary CT Cohort. JACC Cardiovasc Imaging 12(7):1392-1400

5. Hoffmann S et al (2010) Tissue Doppler echocardiography reveals distinct patterns of impaired myocardial velocities in different degrees of coronary artery disease. Eur J Echocardiogr. 11(6):544-549

6. Hoffmann S et al (2012) Tissue Doppler echocardiography improves the diagnosis of coronary artery stenosis in stable angina pectoris. Eur Heart J Cardiovasc Imaging 13(9):724-729

7. Hwang SJ et al (2014) Implications of coronary artery disease in heart failure with preserved ejection fraction. J Am Coll Cardiol 63(25 Part A):2817-2827

8. Borlaug BA et al (2014) The pathophysiology of heart failure with preserved ejection fraction. Nat Rev Cardiol 11:507

9. Drew BJ et al (2010) Prevention of torsade de pointes in hospital settings: a scientific statement from the American Heart Association and the American College of Cardiology Foundation. Circulation 121(8):1047-1060

10. Benoit SR et al (2005) Risk factors for prolonged QTc among US adults: Third National Health and Nutrition Examination Survey. Eur J Cardiovas Prev Rehabil. 12(4):363-368

11. Leren IS et al (2015) Cardiac Mechanical Alterations and Genotype Specific Differences in Subjects with Long QT Syndrome. JACC Cardiovasc Imaging 8(5):501-510
12. Hasenfuss $G$ et al (1999) Relationship between $\mathrm{Na}^{+}-\mathrm{Ca}^{2+}$-exchanger protein levels and diastolic function of failing human myocardium. Circulation 99(5):641-648

13. Vyas $\mathrm{H}$ et al (2008) Mechanical dysfunction in extreme QT prolongation. J Am Soc Echocardiogr. 21(5):511.e15-517.e15

14. Wilcox JE et al (2011) Usefulness of electrocardiographic QT interval to predict left ventricular diastolic dysfunction. Am J Cardiol. 108(12):1760-1766

15. Sauer A et al (2012) Diastolic electromechanical coupling: association of the ECG T-peak to T-end interval with echocardiographic markers of diastolic dysfunction. Circ Arrhythm Electrophysiol 5(3):537-543

16. Namdar M et al (2013) A novel electrocardiographic index for the diagnosis of diastolic dysfunction. PLoS ONE 8(11):e79152

17. Sengupta pp. et al (2018) Prediction of abnormal myocardial relaxation from signal processed surface ECG. J Am Coll Cardiol. 71(15):1650-1660

18. Morise AP, Diamond GA (1995) Comparison of the sensitivity and specificity of exercise electrocardiography in biased and unbiased populations of men and women. Am Heart J 130(4):741-747

19. Heijenbrok-Kal MH, Fleischmann KE, Hunink MG (2007) Stress echocardiography, stress single-photon-emission computed tomography and electron beam computed tomography for the assessment of coronary artery disease: a meta-analysis of diagnostic performance. Am Heart J 154(3):415-423

20. Arbab-Zadeh A, Miller JM, Rochitte CE et al (2012) Diagnostic accuracy of computed tomography coronary angiography according to pre-test probability of coronary artery disease and severity of coronary arterial calcification. The CORE-64 (Coronary Artery Evaluation Using 64-Row Multidetector Computed Tomography Angiography) International Multicenter Study. J Am Coll Cardiol 59(4):379-387

21. De Jong MC, Genders TS, Van Geuns RJ et al (2012) Diagnostic performance of stress myocardial perfusion imaging for coronary artery disease: a systematic review and meta-analysis. Eur Radiol 22(9):1881-1895

22. Jaarsma C, Leiner T, Bekkers SC et al (2012) Diagnostic performance of noninvasive myocardial perfusion imaging using single-photon emission computed tomography, cardiac magnetic resonance, and positron emission tomography imaging for the detection of obstructive coronary artery disease: a meta-analysis. J Am Col Cardiol 59(19):1719-1728

Publisher's Note Springer Nature remains neutral with regard to jurisdictional claims in published maps and institutional affiliations. 